\title{
La política de la industria láctea en Australia
}

DOI: $10.32870 /$ mycp.v8i25.244

Ramón Robledo Padilla*

\section{Introducción}

$\mathrm{E}$ 1 presente artículo trata sobre las políticas de desregulación de la industria de leche que se han realizado en Australia, particularmente los cambios que han ocurrido en los últimos años y que forman parte de una estrategia general de liberalización económica del país. El artículo también se refiere al periodo relativamente corto en que Australia ha realizado dichos cambios, por un lado, con el fin de cumplir con los compromisos que el país había asumido en el marco de la Ronda de Uruguay y, por otro lado, como parte de una estrategia política para presionar a algunas economías desarrolladas para que aceleraran los procesos de liberación en sus respectivos sectores agrícolas; sin embargo, al parecer esto último no ha tenido ningún impacto importante y los países desarrollados continúan subsidiando fuertemente este sector.

\section{Antecedentes}

Australia se caracteriza por ser uno de los países más competentes en la producción de leche a escala mundial; esta característica se debe principalmente a sus condiciones naturales, que le permiten

* Profesor investigador del Departamento de Estudios del Pacífico de la Universidad de Guadalajara. operar con costos de producción muy bajos. Las condiciones naturales que hacen de Australia un país muy competitivo en el ámbito internacional en la producción de leche corresponden a la gran calidad de pastos naturales que son utilizados como principal alimento del ganado, lo que permite reducir los costos y aumentar la cantidad de leche producida. La eficiencia en los costos de producción también es resultado del desarrollo tecnológico, las investigaciones llevadas a cabo para mejorar aún más la producción de pastos, y el mejoramiento genético. ${ }^{1}$

Australia, junto con Nueva Zelanda, es un caso excepcional cuyo sistema de producción de leche, basado en el pastoreo, le permite obtener los costos de producción más bajos dentro de los países desarrollados. Su competitividad se basa en mayor medida en sus ventajas comparativas y en menor medida en la intervención de las políticas gubernamentales. En los casos de la Unión Europea y los Estados Unidos, quienes también son competitivos en la producción de leche, la competitividad se logra principalmente por la intervención de políticas gubernamentales en sus respectivos sectores productivos. Por lo tanto, podemos decir que la competitividad se percibe como el resultado combinado de los efectos de las distorsiones de mercado (provocadas tanto por las políticas como por la competencia imperfecta) y las ventajas comparativas. $^{2}$ 
Importancia del sector lácteo en Australia

En términos del valor bruto de la producción agropecuaria en su conjunto, la producción de leche participa aproximadamente con 10\% (en el año 2004, el valor bruto de la producción agropecuaria en términos reales fue de poco más de 33 mil millones de dólares australianos, aproximadamente 24 mil millones de dólares estadounidenses); sin embargo, la industria de la leche, en su conjunto, ${ }^{3}$ participa con menos del $1 \%$ del PIB nacional. Como se puede apreciar, la participación de la industria de la leche con respecto al PIB total del país no es muy significativa; sin embargo, la importancia de esta actividad se debe a que los precios de sus productos son muy competitivos a escala mundial y sus exportaciones superan el 50\% de su producción interna, lo que en el plano internacional coloca a Australia como el tercer exportador de lácteos más importante del mundo, seguido de la Unión Europea y Nueva Zelanda, en ese orden.

Como se señaló, el precio de la leche en Australia es uno de los más bajos en el mundo dentro de los países desarrollados; esto es debido en gran medida a su sistema de producción de leche basado en el pastoreo, que permite disminuir de manera importante los costos de producción.

En el caso de las economías desarrolladas, la diferencia en el precio pagado al productor es muy irregular; sin embargo, se puede apreciar con claridad que esta variación en el precio con respecto a Australia es desde el 30\% hasta el 100\% más alto. En algunos casos, como el de Japón, la diferencia en el precio es de casi 300\%.

Con respecto a países subdesarrollados, solamente en Uruguay el precio pagado al productor es claramente inferior al que se paga en Australia; en el caso de Argentina es muy similar y en Chile es ligeramente superior.

Cuadro 1

Precio pagado al productor por litro de leche (centavos de dólar estadounidense)

\begin{tabular}{lccccccc}
\hline & 1998 & 1999 & 2000 & 2001 & 2002 & 2003 & 2004 \\
\hline Australia & 17 & 17 & 14 & 14 & 17 & n.d. & n.d. \\
Nueva Zelanda & 14 & 15 & 13 & 17 & 20 & 17 & 22 \\
Estados Unidos & 34 & 31 & 27 & 33 & 26 & n.d. & n.d. \\
Japón & 63 & 72 & 75 & 67 & 65 & n.d. & n.d. \\
Canadá & 34 & 34 & 36 & 35 & 35 & n.d. & n.d. \\
Reino Unido & 31 & 28 & 24 & 23 & 25 & 29 & 33 \\
Uruguay & 13 & 11 & 12 & 12 & 10 & 13 & 15 \\
Chile & 20 & 18 & 19 & 18 & 14 & n.d. & 16 \\
Argentina & 17 & 14 & 15 & 15 & 9 & n.d. & n.d. \\
México & 31 & 31 & 33 & 34 & 32 & 29 & 31 \\
\hline
\end{tabular}

n.d. = no disponible.

Fuente: http://www.fao.org para los datos de 1998 a 2002. Para los demás datos:

http://www.mgap.gub.uy/opypa/ANUARIOS/Anuario05/CadenasProductivas/prod\%20leche.pdf http://statistics.defra.gov.uk/esg/statnot/prmlkpn.pdf., http://www.ganadería.com.mx y

http://www.puc.cl/agronomia/d_investigacion/Proyectos/ProyectosTitulos/pdf/EconomiaAgraria/ JuanlgnacioSilva.pdf. 
Apoyos al sector lácteo en países desarrollados

En la mayoría de los países desarrollados, con excepción de Australia y Nueva Zelanda, existe una gran variedad de mecanismos de apoyo a la producción agropecuaria (incluyen los de la leche). Tal es el caso de algunas de las economías más avanzadas, como las de Europa y los Estados Unidos. Los apoyos son muy diversos, comprenden desde subsidios a la exportación y a los diferentes insumos para la producción hasta restricción a las importaciones, fijación de precios que garantizan la obtención de un beneficio positivo, etc. Como mencioné, a diferencia de otros países desarrollados, Australia y Nueva Zelanda son países desarrollados que mantienen en la actualidad una política de apoyos muy bajos hacia el sector agrícola. El único país que, en menor proporción, apoya al sector agropecuario es Nueva Zelanda. Sin embargo, Australia es el país que más se acerca en cuanto al comportamiento de sus políticas agrícolas. Así lo muestran las reformas que ha puesto en marcha en los últimos años el gobierno australiano, el cual se orienta hacia una política de menor intervención en el sector agropecuario. ${ }^{4}$

Los bajos costos de producción que ha logrado Australia en la mayoría de sus productos agropecuarios le han permitido convertirse hoy en día en fuerte promotor de la eliminación de las distorsiones al comercio internacional provocadas principalmente por países desarrollados, en apego a los lineamientos de la Organización Mundial del Comercio (OMC). Es precisamente este interés por tratar de desregular el sector agrícola en el menor tiempo posible lo que en gran medida permitió el surgimiento en 1986 del grupo Cairns, presidido por Australia. En la actualidad, las diecisiete naciones que integran el grupo Cairns son: Argentina, Brasil, Colombia, Guatemala, Nueva Zelanda, Sudáfrica, Australia, Canadá, Costa Rica, Indonesia, Paraguay, Tailandia, Bolivia, Chile, Malasia, Filipinas y Uruguay. Este grupo de naciones participa en la actualidad con una quinta parte de las exportaciones agrícolas mundiales. De ahí la importancia para ellas de que el mercado agrícola sea liberado. El grupo Cairns ha adquirido tal importancia que actualmente es considerada la tercera fuerza en las negociaciones comerciales, después de los Estados Unidos y la Unión Europea.

No obstante el gran esfuerzo realizado por estos países en la promoción de desregulación del comercio agrícola, la intervención en este sector por parte de algunos gobiernos de países desarrollados sigue siendo muy importante.

\section{Políticas agropecuarias en Australia}

Australia, al igual que la mayoría de los países de todo el mundo, ha realizado cambios en su política económica interna en las últimas dos décadas. Estos cambios están orientados a reducir las intervenciones del gobierno y permitir que las actividades económicas se orienten únicamente por las señales del mercado.

De acuerdo con esta nueva realidad, en los años noventa Australia promovió 
un paquete de políticas orientadas a incrementar los beneficios de los trabajadores del campo y mejorar la competitividad en el ámbito internacional. ${ }^{5}$ En general, los apoyos proporcionados al sector rural antes de 1982 no fueron uniformes. Los más altos niveles de protección fueron dirigidos a la irrigación de cosechas, el sector lácteo, el de azúcar y el de huevo, mientras que otras actividades que eran consideradas más eficientes, como la lana y la carne de vaca, se mantuvieron sin intervención o con un nivel de apoyo relativamente bajo. ${ }^{6}$

La transición que se dio en Australia, entre 1982-1992, estuvo orientada a responder a las señales del mercado y a la eliminación de las intervenciones del gobierno en forma gradual. Se lograron acuerdos entre el gobierno, la industria y el sector laboral para establecer salarios por abajo de la inflación, con el fin de reducir los costos del trabajo y estimular la inversión; al mismo tiempo, el gobierno promovió la desregulación de los mercados financieros, para que estos recursos estuvieran disponibles con mayor rapidez para los individuos y sus negocios. ${ }^{7}$

En este conjunto de reformas, la agricultura fue tratada como parte integral de la economía, sin ninguna consideración especial. Las medidas se orientaron a reducir los niveles de protección comerciales sobre las importaciones, con el fin de ir reduciendo en forma gradual las barreras al comercio.

En 1995, una gran cantidad de productos quedó libre de restricción; únicamente permanecieron con protección algunas industrias agrícolas, como los cítricos, la leche, el azúcar, las frutas y otros, en los cuales la reducción en la protección se ha producido en forma gradual. ${ }^{8} \mathrm{El}$ apoyo al sector lácteo y la desregulación paulatina se deben a que muchos de los países desa- rrollados mantienen altos subsidios y ello provoca que no se realice una competencia equitativa. En el caso de la leche, en 2003, el porcentaje del equivalente del subsidio al productor (PSE) $)^{9}$ en Australia fue 15\%, en Canadá 59\%, en la Unión Europea 51\%, en Japón 77\%, en los Estados Unidos 45\% y en Nueva Zelanda $1 \% .^{10}$

\section{Reforma en la política de la industria de la leche}

Hasta principios de los años ochenta, en Australia existía un control centralizado en el mercado de las exportaciones. Además, debido a que la industria de la leche no era muy grande en términos relativos y estaba formada por pequeñas cooperativas, la obtención de información sobre los mercados de exportación era muy costosa.

Dado lo anterior, el sector procesador fue regulado conjuntamente a través de acuerdos de exportación entre las pequeñas cooperativas productoras de leche, con precios asegurados; además de esto, el precio interno en Australia era controlado por la legislación de cada uno de los estados.

Antes del primero de julio de 2000, la regulación en la industria de la leche de Australia se dividía en dos partes: por un lado estaba el sector de leche líquida, conocido como mercado de la leche (market milk), y por el otro el sector de leche industrializada (manufacturing milk). Ambos sectores fueron apoyados de manera distinta. El mercado de leche líquida fue apoyado en todos los estados de Australia, cada uno de manera independiente, y el sector de leche industrializada consistió en un esquema federal asistido por la Commonwealth.

Los controles de los gobiernos estatales sobre el precio y la oferta de la leche líquida 
conocida como mercado de leche se habían llevado a cabo por lo menos desde 1980 en la mayoría de los estados, y en algunos otros desde antes. El precio que el Estado garantizaba a cierta cantidad de leche líquida estaba muy por encima del precio promedio que se pagaba para el resto de la oferta de leche no regulada, ${ }^{11}$ a pesar de que no existían diferencias en el producto. De acuerdo con la política de desreglar el mercado, las cuotas y los precios de control estatal finalizaron el primero de julio de 2000; esto significa que ya no existen controles sobre la cantidad o el precio de la leche producida y, por tanto, existe un mercado libre en las ventas de leche líquida en Australia. ${ }^{12}$

En lo que concierne al apoyo por parte del gobierno de la Commonwealth, dos eran los programas más importantes que ofrecían asistencia al sector. Los programas se conocieron como el Plan Kerin y el Plan Crean, de 1986 y 1992, respectivamente.

La estrategia del Plan Kerin se basaba en una política que mantenía los precios internos de los productos lácteos por arriba de los precios de exportación (aproximadamente dos centavos por litro), esto estaba ligado a un pago que se hacía para apoyar las exportaciones de productos lácteos: el dinero utilizado para estos fines provenía de un impuesto que se aplicaba a toda la producción de leche en Australia. Esta estrategia, que estimulaba el incremento de los precios internos de los lácteos, finalmente se traducía en un beneficio neto hacia los productores de leche, ya que se incrementaba el precio de la leche que era utilizada para la elaboración de productos lácteos destinados para consumo interno. ${ }^{13}$ El Plan Crean, sustituyó al anterior, y lo que planteó básicamente fue ir reduciendo los apoyos gradualmente, hasta eliminarlos en junio 30 de 2000.
A partir de 1995 los apoyos tuvieron que ser rediseñados, debido a que, de acuerdo con la OMc, el esquema de pagos para apoyar la exportación de productos lácteos constituía un subsidio a las exportaciones.

El nuevo esquema fue administrado por la Corporación Australiana de Lácteos (Australian Dairy Corporation, ADC); asimismo, las medidas fueron derivadas de los planes Kerin y Creen, los cuales, como se mencionó, fueron introducidos en 1986 y 1992, respectivamente. A partir de julio de 1995 se estableció esta nueva estrategia política, con el propósito de cumplir los acuerdos que Australia había contraído en el marco de la Ronda de Uruguay. Para esto, el nuevo plan buscó que los industriales de la leche mantuvieran los mismos beneficios que con el plan anterior. El cambio fundamental con respecto a los planes anteriores consistía básicamente en que el apoyo interno se realizara de tal modo que no se interpretara como un subsidio a las exportaciones. Finalmente, la modificación quedó de la siguiente manera: los productores de leche pagaban un impuesto por la leche que vendían internamente para consumo directo (alrededor de dos centavos por litro); a su vez, los industriales de la leche pagaban un impuesto (en 1996-1997 el pago fue de alrededor de cuatro centavos por litro) por los productos lácteos que se vendían internamente. La leche utilizada para la elaboración de lácteos destinados a la exportación quedó exenta de impuestos. En este sentido, los apoyos a las exportaciones bajo esta lógica terminaron en junio 30 de 2000. Finalmente, los recursos de los impuestos que habían sido obtenidos mediante estas nuevas medidas fueron utilizados para realizar pagos a aquellos productores cuya leche era destinada a la elaboración de productos lácteos, ${ }^{14} \mathrm{y}$ 
los criterios para realizar dichos pagos se establecieron en función de la calidad y la cantidad de la leche ofrecida.

De 1986 a 1996, la ayuda a la industria de leche había declinado aproximadamente en $65 \%$ y se esperaba que fuera eliminada completamente, en forma gradual para el año 2000. No obstante la reducción de los apoyos por parte del gobierno desde 1986, una década después la producción de leche se había incrementado en una tercera parte, y el volumen de las exportaciones había aumentado al doble, a pesar de que hubo una reducción del 10\% en el número de cabezas de ganado y una disminución del $30 \%$ de las granjas lecheras. ${ }^{15}$

Para aminorar los efectos de la desregulación en el sector de la industria de leche, el gobierno australiano introdujo una nueva legislación que comenzó a aplicarse el primero de julio del año 2000. Esta nueva ley se conoce como Paquete de Ajustes a la Industria Láctea (Dairy Industry Adjustment Package, DIAP), que comprende tres programas. ${ }^{16} \mathrm{El}$ primero consiste en financiar mediante pagos trimestrales durante los próximos ocho años, a partir del 2000, a todos aquellos granjeros que estuvieran interesados en continuar la producción de leche. Para realizar estos pagos se tomaron como referencia los volúmenes de producción de leche líquida e industrializada durante los años 1998-1999.

El segundo programa consistió en apoyar con 45,000 dólares australianos (equivalentes a 25,875 dólares estadounidenses), libres de impuestos, aquellos granjeros que desearan abandonar la actividad. Finalmente, el tercer programa tuvo como objetivo destinar recursos a las comunidades dependientes del sector lechero que fueron afectadas por los programas de desregulación.

\section{Conclusión}

Los procesos de apertura comercial y desregulación económica que se han dado en la economía australiana, y en particular en la industria de la leche, no han mermado la producción ni la productividad de la industria. Ello se debe a que los australianos, aprovechando sus condiciones naturales, dentro del ámbito de los países desarrollados, tienen un sistema de producción muy eficiente en términos de los costos de producción, además de otras características como su vocación, organización e inversión en investigación científica encaminada a mejorar la productividad de la industria. $\mathrm{Al}$ parecer, con las medidas que se han tomado en los últimos años es probable que continúe el proceso de centralización de la industria de la leche, como se ha observado hasta el momento. my

\section{Notas}

1 Cfr. Australian Dairy Corporation, Annual Report, p. 5.

2 Velázquez, Víctor Manuel, p. 81.

3 La industria de la leche comprende tanto la actividad primaria por medio de la cual se obtiene la materia prima (leche) como la actividad secundaria, que incluye los procesos de transformación que se utilizan para la elaboración de derivados lácteos.

4 Cfr. 105 World Bank Discussion, p. 8.

5 http://www.dpie.gov.au septiembre de 1997, p. 3.

6 Op. cit. http://www.dpie.gov.au, pp. 6 y 7.

7 Op. cit. http://www.dpie.gov.au, p. 7.

8 Op. cit. http://www.dpie.gov.au, pp. 9 y 10.

9 El subsidio equivalente al productor es un indicador del valor de las transferencias monetarias a la agricultura que resulten de las políticas agrícolas en un año dado. En este caso, por ejemplo, a los productores de leche australianos se les transfirió el 15\% del valor total de la producción de leche en el año 2003.

10 OECD, 2004.

11 En la década de los noventa, el precio más bajo de la leche fluida controlada fue de 48 centavos australianos, y el más alto de 55 centavos. En el mismo periodo, el precio promedio de la leche no 
controlada fue de entre 20 y 28 centavos como promedio.

12 http://www.aph.gov.au/library/pubs/bd/200001/01bd167.htm.

13 http:www.dairy.com.au./adc/profile/markarr, p. 1. (mayo 1997).

14 Ibíd., p. 2, y http://www.dairy.com.au/adc/profile/dairystr, p. 2 (mayo de 1997).

15 http://www.dpie.gov.au/dpie/agriculture/agricultural_reform.html, p 12 (mayo de 1997).

16 ABARE, pp. 4-5.

\section{Bibliografía}

ABARE, The Australian Bureau of Agricultural and Resource Economics (2001), The Australian Dairy Industry, Impact of an open market in fluid milk supply, Report to the Federal Minister For Agriculture, Fisheries and Forestry, enero.
Australian Dairy Corporation, Annual Report 19891990.

Australian Dairy Industry Council (1996), The comprehensive reference to the Australian Dairy Industry, Morescope Publishing Pty Ltd.

Muñoz Rodríguez, Manrrubio y Odermatt Pius (1991), El sistema leche de México en el marco del Tratado Trilateral de Libre Comercio, Universidad Autónoma de Chapingo, noviembre.

OECD (2004), Agricultural Policies in OECD Countries at a Glance.

Velázquez, Víctor Manuel, Roberto García M. y Miguel Martínez D. (1998), Análisis comparativo de los costos de producción de leche de vaca en siete países, en Políticas Agrícolas, vol. III, núm. 3.

World Bank Discussion Papers 105 (1993), Redefining the Role of Government in Agriculture for the 1990s, Odin Knudsen, John Nash, with contributions by James Bovard, Bruce Gardner, L. Alan Winters, Washington, segunda impresión, agosto. 